Article

\title{
Typical Damage Prediction and Reliability Analysis of Superheater Tubes in Power Station Boilers Based on Multisource Data Analysis
}

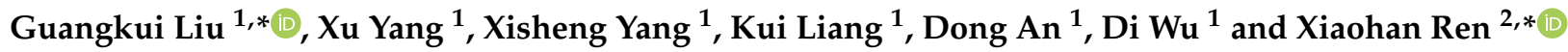 \\ 1 China Special Equipment Inspection \& Research Institute, Beijing 100029, China; yangxu@csei.org.cn (X.Y.); \\ yangxisheng@csei.org.cn (X.Y.); liang2482@sina.com (K.L.); andongfun@163.com (D.A.); \\ wudi@csei.org.cn (D.W.) \\ 2 Institute of Thermal Science and Technology, Shandong University, Jinan 250061, China \\ * Correspondence: liuguangkui9527@163.com (G.L.); xiaohan09126@gmail.com (X.R.); \\ Tel.: +86-138-1023-4549 (G.L.); +86-133-5669-8944 (X.R.)
}

check for updates

Citation: Liu, G.; Yang, X.; Yang, X.; Liang, K.; An, D.; Wu, D.; Ren, $X$. Typical Damage Prediction and Reliability Analysis of Superheater Tubes in Power Station Boilers Based on Multisource Data Analysis. Energies 2022, 15, 1005. https:// doi.org/10.3390/en15031005

Academic Editors: Francisco Vera García and Francisco P. Brito

Received: 26 October 2021

Accepted: 12 January 2022

Published: 29 January 2022

Publisher's Note: MDPI stays neutral with regard to jurisdictional claims in published maps and institutional affiliations.

Copyright: (c) 2022 by the authors. Licensee MDPI, Basel, Switzerland. This article is an open access article distributed under the terms and conditions of the Creative Commons Attribution (CC BY) license (https:// creativecommons.org/licenses/by/ $4.0 /)$.

\begin{abstract}
The superheater and re-heater piping components in supercritical thermal power units are prone to creep and fatigue failure fracture after extensive use due to the high pressure and temperature environment. Therefore, safety assessment for superheaters and re-heaters in such an environment is critical. However, the actual service operation data is frequently insufficient, resulting in low accuracy of the safety assessment. Based on such problems, in order to address the issues of susceptibility of superheater and re-heater piping components to creep, inaccurate fatigue failure fracture, and creep-fatigue coupling rupture in a safety assessment, their remaining life prediction and reliability, as well as the lack of actual service operation data, multisource heterogeneous data generated from actual service of power plants combined with deep learning technology was used in this paper. As such, three real-time operating conditions' data (temperature, pressure, and stress amplitude) during equipment operation are predicted by training a deep learning architecture long short-term memory (LSTM) neural network suitable for processing time-series data and a backpropagation through time (BPTT) algorithm is used to optimize the model and compared with the actual physical model. Damage assessment and life prediction of final superheater tubes of power station boilers are carried out. The Weibull distribution model is used to obtain the trend of cumulative failure risk change and assess and predict the safety condition of the overall system of pressurized components of power station boilers.
\end{abstract}

Keywords: superheater and re-heater; creep and fatigue failure fracture; safety assessment; reliability; deep learning; Weibull distribution

\section{Introduction}

Although nuclear power, hydroelectric power, wind power, solar energy, and other energy-acquiring power generation technologies have developed rapidly, the world's electricity supply is still dominated by thermal power and improving steam parameters of thermal power units is an effective solution to improve power generation efficiency and alleviate the energy crisis and environmental damage existing at present $[1,2]$. The long-term service of high-temperature materials in power plant boilers usually leads to different degrees of creep, fatigue, and creep-fatigue coupling damage under the coupling of extreme conditions, such as high temperature, high pressure, and stress fluctuations, which cause potential problems in the safe operation of the unit. However, a method for rapidly and accurately assessing damage status and the remaining life of a component is lacking at this stage.

Process control engineering continuously stores and accumulates not only these process operation data in time but also extends data transmitted between and within collected 
devices and people in space to obtain large-capacity data at different scales in both time and space dimensions. However, such massive amounts of data are poorly applied to the process of equipment health diagnosis and damage assessment. According to [3], the process industry can improve productivity using big data analysis and applications. A model was established using the process's normal operation data. Based on such a model, the fault detection indicators and their control limits for fault detection could be defined for fault detection and diagnosis. The cause of faults can be detected before product quality control and user feedback occur. In addition, abnormal working conditions, which could lead to serious faults and unsafe accidents, can be diagnosed. It is impossible to describe the dynamics of the process when process data are dynamic time series with strong autocorrelation or dynamic correlation with traditional static latent structure modeling methods. Thus, providing an accurate quantitative assessment of the damage state of in-service equipment is also impossible.

With the rapid development of artificial intelligence technology, deep learning provides strong technical support for health diagnosis and analysis prediction of industrial equipment big data due to its powerful data processing and analysis capability $[4,5]$. Since 2006, deep learning has become an important research direction that redefines state-of-theart performance in a wide range of areas, such as object recognition, image segmentation, speech recognition, and machine translation [6-12]. Data-driven solutions that use deep learning have been increasingly used with the widespread deployment of low-cost sensors as a source of data in modern manufacturing systems and connectivity to the Internet. These sensors continuously collect measurement data in time series, and patterns found in time series data allow for proactive anomaly detection and trend prediction to prevent failures and defects [6]. Deep learning algorithms demonstrate excellent performance in many areas $[11,12]$. Although deep learning research has been extensively applied to machine health monitoring, studies on data pooling for remaining life expectancy prediction are limited. Accurate remaining life prediction can remarkably improve the reliability and operational safety of industrial components or systems, avoid fatal failures, and reduce maintenance costs, especially in the remaining life prediction of large equipment [13-17].

Heating surface systems in supercritical thermal power units, especially final-stage superheater tubes, are exposed to the most severe environment and prone to creep and fatigue failure fracture during prolonged operation under high-pressure and -temperature environments. Hence, the accurate prediction of creep and fatigue damage life is important in the use and design of final-stage superheater tubes. A series of studies on deep learning in industrial big data time prediction has obtained excellent results in different scenarios for the prediction of the remaining lifetime of equipment components [18-26].

The direction of creep, fatigue, and coupled creep-fatigue damage characteristics of key pressure-bearing component materials of power plant boilers and their remaining life prediction and early warning of pressure-bearing components were explored in this study using multisource heterogeneous data generated from the actual service of power plants combined with deep learning technology. We divided the life and damage prediction into the following parts: (1) autoregressive industrial time-series data and (2) damage and life prediction problems. The deep learning architecture of long short-term memory (LSTM) [11] is suitable for processing temporal data and applied to train temporal prediction models using run-time data. Then the models are optimized using the backpropagation through time (BPTT) algorithm. Once the models are obtained from the final training, they are used to predict three types of real-time operating condition data, such as temperature, pressure, and stress amplitude, during equipment operation. Meanwhile, predicted operating condition data are used as input for damage and life calculations with a physical model to improve the results and provide a new method for damage assessment and life prediction of high-temperature components. 


\section{Simulation and Calculation Methods}

\subsection{Creep-Fatigue Interaction of Materials}

The case where the material is simultaneously subjected to alternating loads, including alternating pressure or temperature, at high temperatures is called creep-fatigue interaction damage, which is a complex damage problem.

Creep-fatigue interaction damage of the material can be expressed as follows:

$$
\mathrm{D}=\mathrm{D}_{\mathrm{f}}+\mathrm{D}_{\mathrm{c}}=\sum_{\mathrm{i}=1}^{\mathrm{n}_{\mathrm{c}}} \frac{\mathrm{t}_{\mathrm{i}}}{\mathrm{T}_{\mathrm{ic}}}+\sum_{\mathrm{j}=1}^{\mathrm{n}_{\mathrm{f}}} \frac{\mathrm{n}_{\mathrm{j}}}{\mathrm{N}_{\mathrm{jf}}}
$$

where $D_{f}$ indicates the fatigue damage fraction, $D_{c}$ indicates the creep damage fraction, and $\mathrm{D}$ indicates the accumulated damage at the time of reaching failure. $\mathrm{T}_{\mathrm{ic}}$ is the load retention time at the ith load level, $\mathrm{n}_{\mathrm{c}}$ is the creep fracture time at the ith load level, $\mathrm{C}$ is the number of stress levels ( $n_{c}=$ shear stress/shear strength), $n_{j}$ is the number of cycles at the $\mathrm{jth}$ stress/strain amplitude, $\mathrm{N}_{\mathrm{jf}}$ is the number of cycles where fatigue failure occurs at the $\mathrm{jth}$ stress/strain amplitude and $\mathrm{n}_{\mathrm{f}}$ is the number of different stress amplitudes [27].

\subsection{Operating Condition Data of Final Superheater Operation}

Creep and fatigue damage will jointly affect the service life of the final superheater heat exchanger tube due to their dual role when constantly exposed to a high-temperature and -pressure environment. Failure of structural components before their predicted lifetimes can often result in significant losses. This study aims to obtain increasingly reliable remaininglife prediction results, learn existing operation rules and laws using deep learning methods, and simulate the future operation trend state of the power plant boiler directly using existing power plant boiler operation data.

\subsection{Model for Simulating Equipment Operating Conditions}

DCS (Distributed Control System, as shown in Figure 1) used in industrial production generate large amounts of data every minute of every day. DCS data collected at different times are called time-series data, which are information with one or more characteristics that change over time.

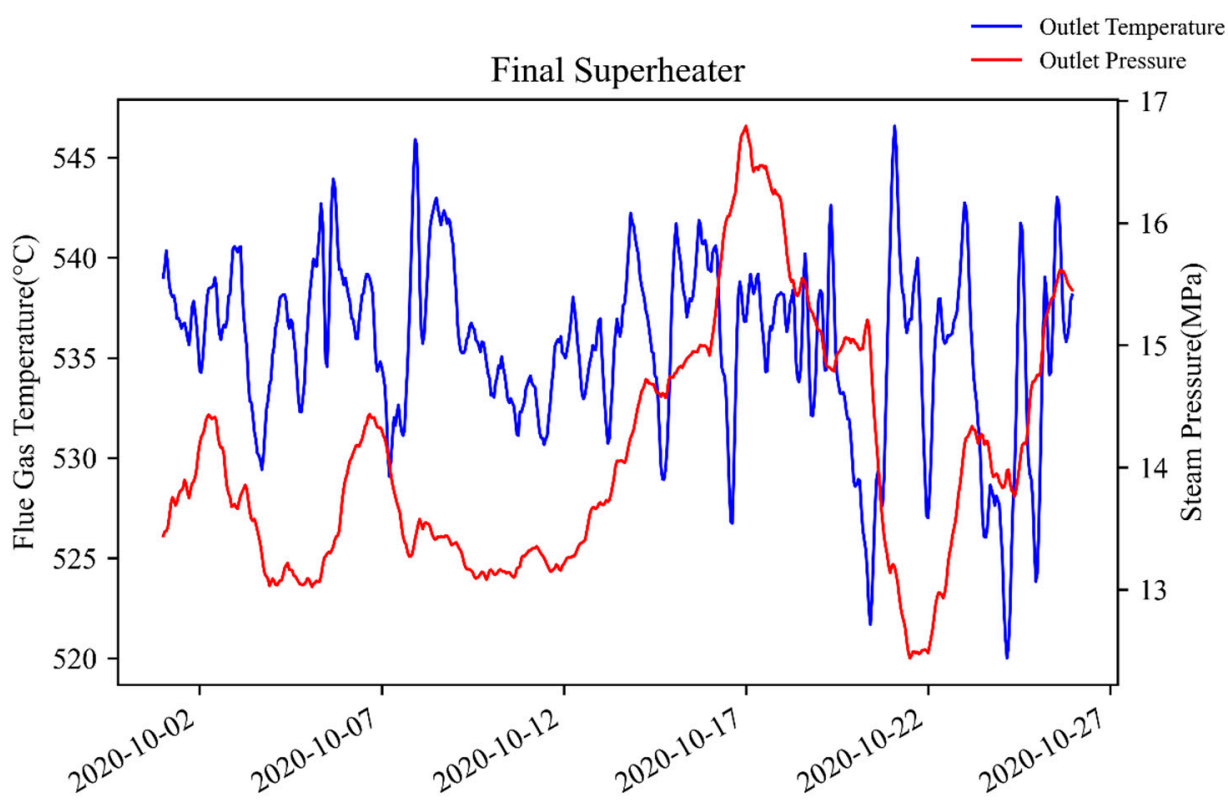

Figure 1. DCS system collects temperature and pressure data from the final superheater outlet.

Time-series forecasting is classified differently depending on the perspective. Timeseries forecasting can be divided into traditional statistics and machine learning (also 
divided into non-deep and deep learning) from the point of view of implementation principles. As shown in Figure 2, single and multistep forecasts are simply the difference between forecasting one time unit and multiple time units in the future. Time-series forecasting can be divided into (1) autoregressive forecasting and forecasting using covariates from the perspective of input variables depending on whether or not the dimension contains covariates, (2) point and probabilistic predictions from the perspective of output results, and (3) univariate, multivariate, and multiple time series predictions from the perspective of the number of targets. These classifications are under different perspectives, and the same algorithm is often only used based on one of the classifications; for example, traditional statistical algorithms are only feasible for autoregressive prediction but unsuitable for covariate prediction [17].

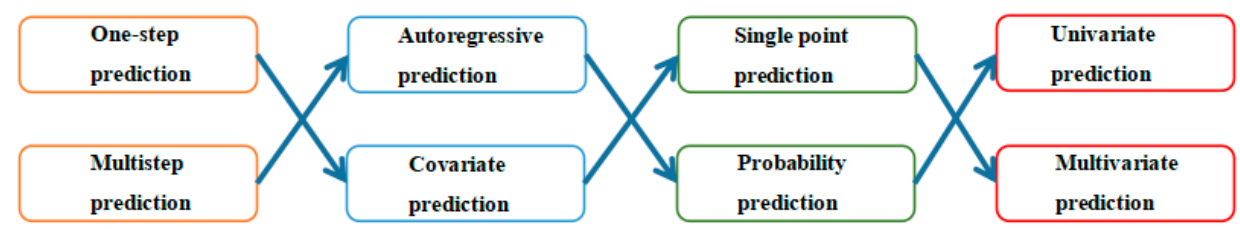

Figure 2. Time-series prediction classification.

As shown in Figure 3, three typical algorithms can be used for timing prediction, of which the LSTM algorithm is the most widely used and best applied. Simple moving average (SMA) is an easy-to-implement algorithm in statistics that is very effective in reflecting trends in data over time while ignoring cyclical changes in data. The ARIMA algorithm is obtained from the moving average (MA) and adding the autoregressive (AR) operator. With the complement of the moving average, it reflects the cyclical pattern of data and predicts the time series in a one-dimensional and time-strong correlation scenario successfully from a statistical point of view.

\section{SMA/ARIMA}

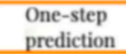

prediction

2. LSTM

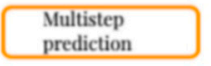

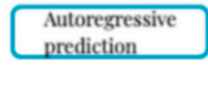

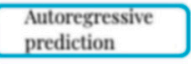

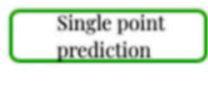

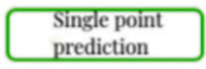

Univariate prediction

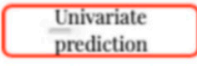

Figure 3. Time-series prediction algorithm.

\subsection{Creep-Fatigue Life Calculation}

Fatigue Life Calculation of Final-Stage Superheater

The fatigue damage model can be used to calculate the accumulated fatigue damage of the final superheater made of Gr. 91 during future service after applying the trained neural network model to predict the pressure and temperature of boiler operation. The fatigue cycle of Gr. 91 steel related to the strain amplitude of the material is expressed as follows:

$$
\frac{\Delta \varepsilon}{2}=0.002066\left(2 \mathrm{~N}_{\mathrm{F}}\right)^{-0.04108}+0.532\left(2 \mathrm{~N}_{\mathrm{F}}\right)^{-0.6682}
$$

where $\Delta \varepsilon$ indicates the dimensionless strain amplitude and $N_{F}$ indicates the fatigue life under strain and is the maximum number of cycles withstood under that strain condition [28].

Circumferential stresses are primarily applied inside the superheater tube. Hence, calculating circumferential stresses on the basis of pressure conditions inside the tube is necessary to determine strain amplitude variables inside the tube and thus obtain strain 
variables. The value of the strain variable is obtained when the working pressure is changed. The circumferential stress is calculated as follows:

$$
\sigma_{\theta}=\frac{p\left(D_{0}-S\right)}{2 S}
$$

where $\sigma_{\theta}$ indicates the circumferential stress, $\mathrm{MPa} ; p$ indicates the pressure under normal operation of the pipeline, $\mathrm{MPa} ; D_{0}$ indicates the outside diameter of the pipe, $\mathrm{mm}$; and $S$ indicates the wall thickness of the pipe, $\mathrm{mm}$.

The strain can be calculated from the stress and modulus of elasticity as follows:

$$
\varepsilon=\frac{\sigma}{E^{\prime}}
$$

where $\varepsilon$ indicates the amount of dimensionless strain change; $\sigma$ indicates the stress, $\mathrm{MPa}$; and $E^{\prime}$ indicates the modulus of elasticity, $\mathrm{MPa} / \mathrm{mm}^{2}$.

\subsection{Pressure Parameter Fluctuation Detection}

Detecting the fluctuation of the pressure parameter is crucial when predicting the fatigue life of a material. Fatigue damage fraction per unit time, total accumulated fatigue damage, and the fatigue remaining life of different materials of various parts of the system can be calculated on the basis of the fluctuation value of the pressure parameter and joint Equations (2)-(4). Actual scenes typically contain many noise points in data, and the selection of the peak position may be biased. Fully understanding parameters, such as width, threshold, distance, and especially prominence, is important to obtain a satisfactory extraction peak because noise may change the position of the local maximum.

Distance represents the minimum horizontal distance $(\geq 1)$ required between adjacent peaks in terms of sample size. The selected distance value in the example is small to ensure that noise points with large values in the leftmost part of simulated data are considered peaks. Prominence represents the prominence of the peak and can be interpreted as the minimum descent height required to move from the summit to any higher terrain. The prominence value selected in Figure 4 is 1 .

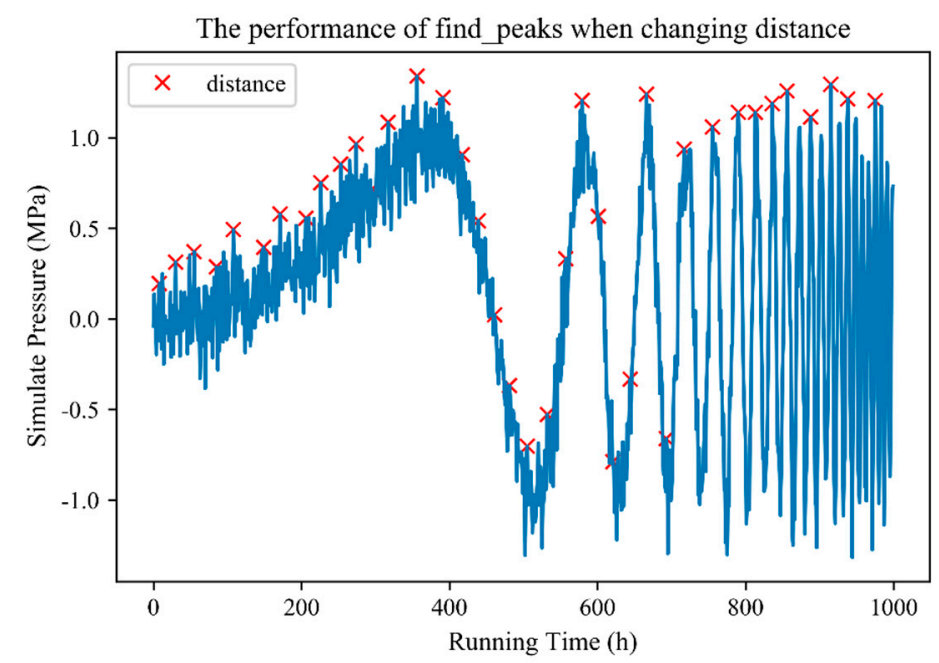

(a)

Figure 4. Cont. 


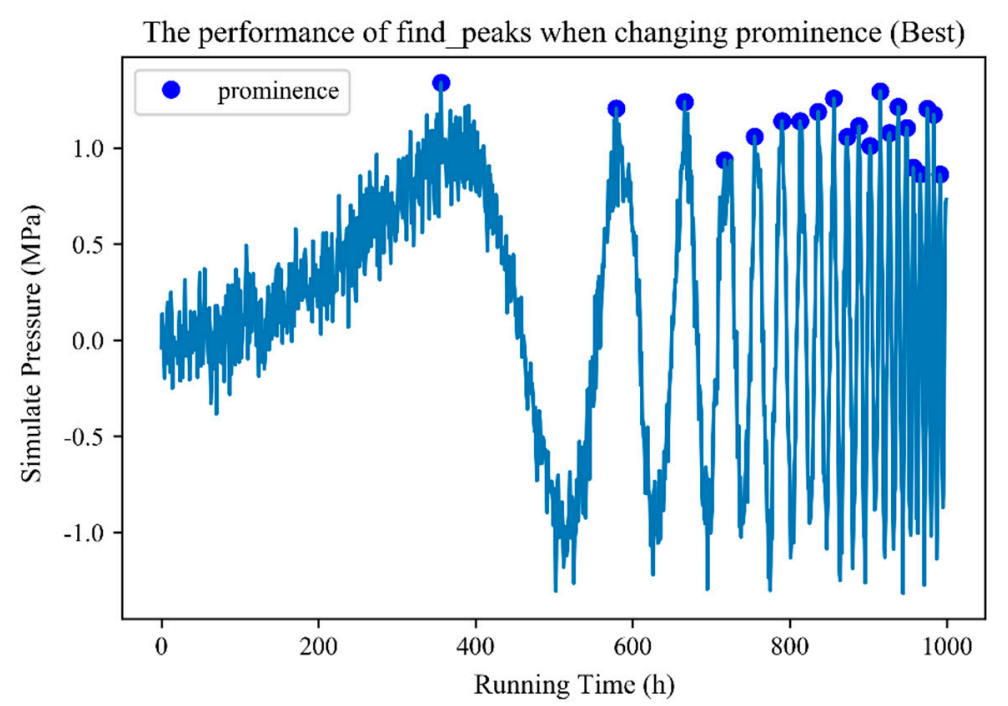

(b)

Figure 4. Diagram of (a) distance- and (b) prominence-based wave detection algorithm to capture peaks in pressure changes.

The threshold represents the difference between the peak and neighboring points. The threshold value selected in Figure 5 is 0.4 . Noise points are typically located near peak points, and the interpolation with peak points fails to reach the range specified by the threshold. Hence, the algorithm fails to search for peak points.

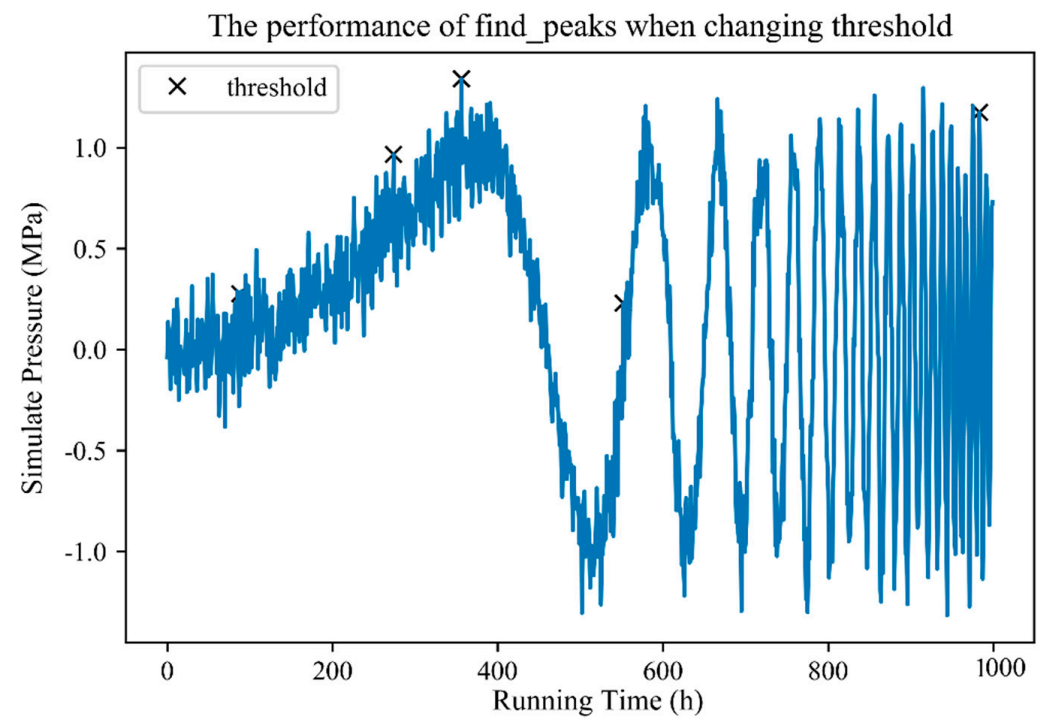

Figure 5. Diagram of the threshold-based wave detection algorithm to capture peaks in pressure changes.

\subsection{Creep Life Calculation of Final-Stage Superheater}

Power plant boilers become supercritical when frequent peaking occurs in the longterm operating temperature which is higher than the temperature at which creep damage occurs in the material. Therefore, the heating surface of the components of the tube still presents different degrees of creep damage regardless of the depth of peaking and the creep damage rate of the material will also show corresponding changes with operating parameter changes. The main parameters include flue gas temperature, flue gas flow rate, steam pressure, and steam temperature. The operating data parameters at the millisecond level for the time period can be obtained and then combined with the material creep life 
assessment model to calculate the creep damage and remaining life of pressurized parts of the heating surface per unit time using real-time DCS operating data collected from the power plant boiler.

The Larson-Miller (L-M) parameter method is based on the principle that a constant $\mathrm{P}$ exists between the service temperature $\mathrm{T}$ of the material and the service life $t$ and is widely used for the creep life calculation of boiler tube materials to obtain improved accuracy. L-M can be expressed as follows [29]:

$$
\mathrm{P}(\sigma)=(\lg t+\mathrm{C}) T
$$

where $C$ indicates the constant of the material, with a value selected with consideration for the composition, organization, and treatment process of the material. The constant $\mathrm{C}$ value of a material is usually solved using linear regression with the results of persistence experiments at and near the working temperature of the selected material at a combination of multiple temperatures (at least three) and stresses (at least four) to solve for the $\mathrm{C}$ value in Equation (5).

$$
\operatorname{Lgt}=\mathrm{C}+\left(\mathrm{C}_{1} \lg \sigma+\mathrm{C}_{2} \lg ^{2} \sigma+\mathrm{C}_{3} \lg ^{3} \sigma+\mathrm{C}_{4} \lg ^{4} \sigma+\ldots+\mathrm{C}_{0}\right) / \mathrm{T}
$$

The $C$ value related to the material is obtained using the regression calculation of Equation (6). The $\mathrm{C}$ value, temperature $\mathrm{T}$, and fracture time $\mathrm{t}$ are substituted into Equation (5) to obtain the $p$-value function corresponding to stress $\sigma$. The $\sigma-\mathrm{P}(\sigma)$ curve is drawn, and the functional relationship between $p$-value and stress $\sigma$ is obtained through the function fitting calculation as follows:

$$
P_{t}=\mathrm{K}_{1}+\mathrm{K}_{2} \lg \sigma
$$

where $\mathrm{K}_{1}$ and $\mathrm{K}_{2}$ are constants.

Therefore, the remaining life $t_{r}$ of the material under constant wall stress $\sigma$ and equivalent temperature $\mathrm{T}$ is the time $\mathrm{t}$ at which the fracture occurs under that stress temperature condition minus the running time $t_{0}$ experienced by the wall; that is, $t_{r}=t-$ $t_{0}$. Combined with Equations (5) and (7), $t_{r}$ can be rewritten as follows:

$$
\mathrm{t}_{\mathrm{r}}=10^{\left(\mathrm{K}_{1}+\mathrm{K}_{2} \lg \sigma\right) / \mathrm{T}-\mathrm{C}}-\mathrm{t}_{0}
$$

According to Equation (8), the remaining life of the material can be predicted for the case of wall stress $\sigma$ and equivalent temperature T. The remaining life of the material can be deduced using Equation (8) when the pipe parameters of the material in service, such as outer diameter, wall thickness, and other dimensional data of the pipe, are known and the equivalent temperature of the material in service $\mathrm{T}$ is calculated.

\subsection{Critical Component Reliability Assessment}

Pressure-bearing components of power station boilers present a complex structure and contain many tube systems. Notably, heating surface components are usually composed of a component system with thousands of tubes of different materials. However, the actual inspection process can only be carried out by sampling the risk state assessment of some heating surface tubes and the overall components are not fully analyzed. The Weibull model is used in this study to assess the overall risk status of the three-stage superheater and high-temperature re-heater, which present the most severe environments for supercritical power plant boilers in service. Hence, the quantitative calculation of the overall risk status of components can also be conducted using small samples (a small number of inspection results).

The distribution function of the three-parameter Weibull distribution is expressed as follows [30,31]:

$$
F(t)=1-\exp \left[-\left(\frac{t-\gamma}{\eta}\right)^{\beta}\right]
$$


where $\eta$ is a scale parameter called characteristic lifetime, which is a lifetime mean that provides the approximate location of the midpoint of the distribution; $\beta$ indicates the shape parameter, with a magnitude that determines the shape of the density profile; $\gamma$ is the location parameter and also known as the minimum life, which indicates that the product will not fail before $\gamma$; and $\eta, \beta>0$.

Reliability characteristic quantities of the three-parameter Weibull distribution are presented as follows.

Fault probability density function:

$$
f(t)=\frac{\beta}{\eta}\left(\frac{t-\gamma}{\eta}\right)^{\beta-1} \exp \left[-\left(\frac{t-\gamma}{\eta}\right)^{\beta}\right]
$$

Reliability function:

$$
R(t)=\exp \left[-\left(\frac{t-\gamma}{\eta}\right)^{\beta}\right]
$$

Unreliability function:

$$
F(t)=1-\exp \left[-\left(\frac{t-\gamma}{\eta}\right)^{\beta}\right]
$$

Failure rate function:

$$
\lambda(t)=\frac{\beta}{\eta}\left(\frac{t-\gamma}{\eta}\right)^{\beta-1}
$$

Average life expectancy:

$$
\theta=\gamma+\eta \Gamma\left(1+\frac{1}{\beta}\right)
$$

where $\Gamma(\bullet)$ indicates the gamma distribution.

Reliable life:

$$
t_{R 0}=\gamma+\eta\left[\ln \left(\frac{1}{R_{0}}\right)\right]^{\frac{1}{\beta}}
$$

The three-parameter Weibull distribution will be transformed into a two-parameter one when $\gamma=0$, at which point probability density and unreliability distribution functions are expressed in the following forms [32]:

$$
\begin{gathered}
f(t)=\frac{\beta}{\eta}\left(\frac{t}{\eta}\right)^{\beta-1} \exp \left[-\left(\frac{t}{\eta}\right)^{\beta}\right] \\
F(t)=1-\exp \left[-\left(\frac{t}{\eta}\right)^{\beta}\right]
\end{gathered}
$$

\section{Results and Discussion}

\subsection{Creep Life Calculation of Final-Stage Superheater}

The results of the neural network training model were used to utilize DCS dynamic data effectively and derive the respective dynamic creep damage and remaining life relationship prediction diagrams for T91 and TP347H materials under different operating conditions in the final superheater stage, as shown in Figures 6 and 7. Both superheater T91 steel and TP347H steel showed fluctuation in the accumulated degree of creep damage with the extension of service time due to the fluctuation of operating parameters of power station boilers in actual service. This finding is significantly different from the linear prediction of traditional physical models using design or historical average parameters. The analysis of actual operating parameters of the real-time call obtained an increasingly accurate assessment of the actual creep damage of superheater pressure-bearing compo- 
nents through the real-time call evaluation of actual operating parameters and improved the accurate diagnosis while avoiding over- or underestimation. The creep damage accumulation caused by T91 steel in the same service time was significantly lower than that by TP347H steel due to the difference in the service environment. However, compared with T91 steel, austenitic TP347H steel presented a lower dislocation density and a slower rate of microstructure aging in service that resulted in a smoother rise in creep rate due to microstructural stability of the material and other reasons. Significant microstructure evolution occurred in the service process due to the high density of dislocations in the microstructure of martensitic T91 steel. Moreover, the special change in the early stage of tissue aging due to slippage of movable dislocations caused significant aging of the tissue, which in turn affected the creep resistance of the T91 material, thereby showing a significant damage transition in the predicted results [33,34]. Notably, in addition to the environmental fluctuation factor, microstructure evolution is also an important reason for the difference in damage accumulation characteristics of the two materials.

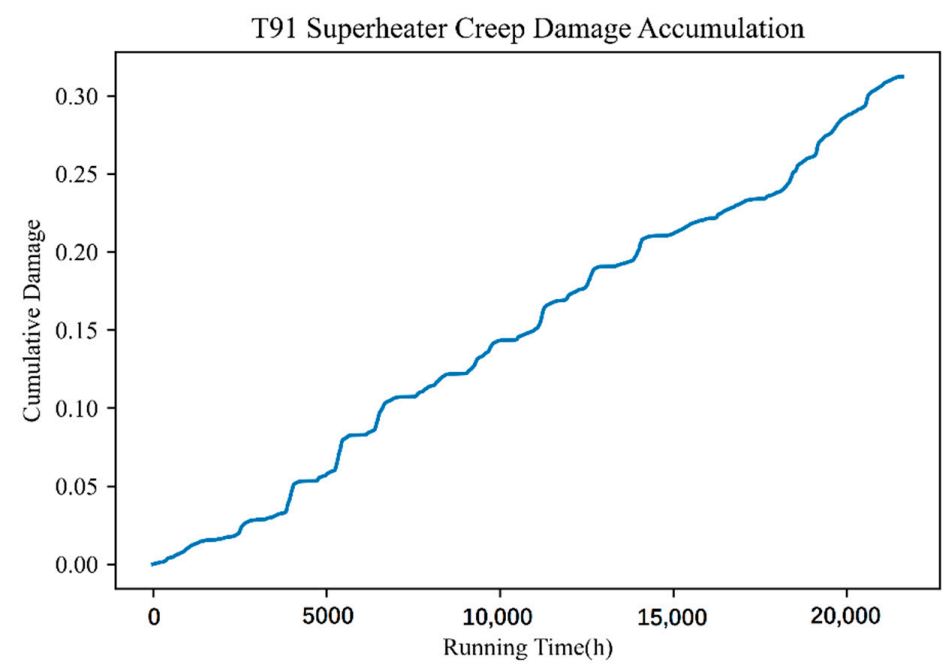

Figure 6. Relationship of service time and creep damage amount of final superheater T91 material.

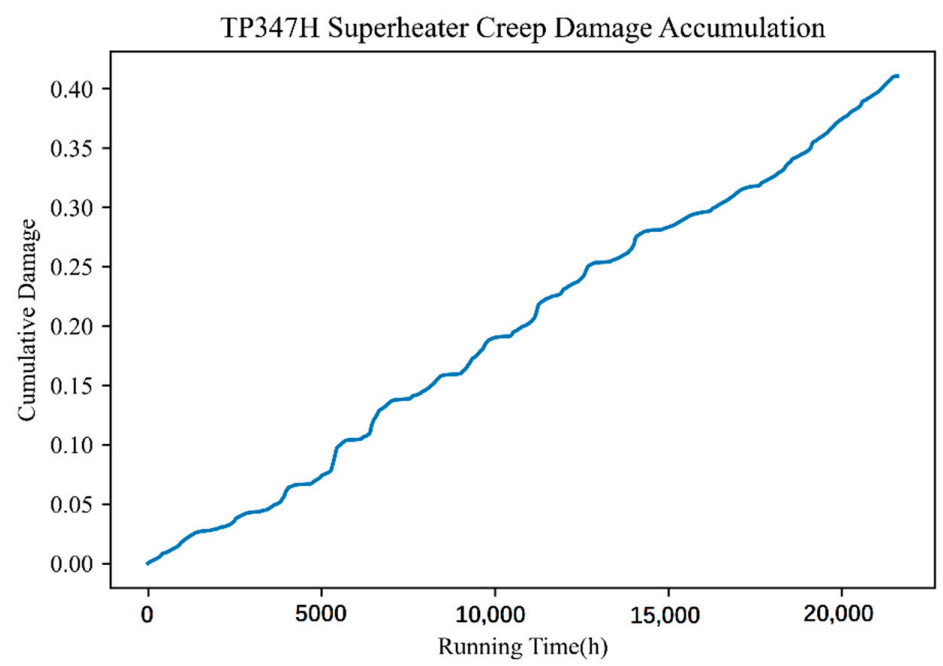

Figure 7. Relationship of service time and creep damage amount of high-temperature superheater TP347H material.

\subsection{Fatigue Life Calculation of Final-Stage Superheater}

Supercritical power plant boilers will produce fatigue damage to the material when frequent deep peaking steam pressure fluctuations occur in the heated surface tube and the operating parameters change (main parameters include flue gas temperature, flue 
gas flow rate, steam pressure, and steam temperature), influencing the rate of fatigue damage to the material, fatigue cycle, and frequency accordingly. The respective fatigue damage and remaining life of pressurized parts of the heating surface in a fixed cycle can be calculated using real-time DCS operational data collected from the power plant boiler combined with the material fatigue life assessment model, as shown in Figures 8 and 9. The sensitivity of fatigue characteristics of T91 and TP347H materials was less than that of creep damage although a relationship existed with the microstructure structure. Thus, the similar fatigue damage accumulation characteristics of the two materials further indicated that the fluctuation of environmental parameters exerts additional influence on the fatigue damage of materials.

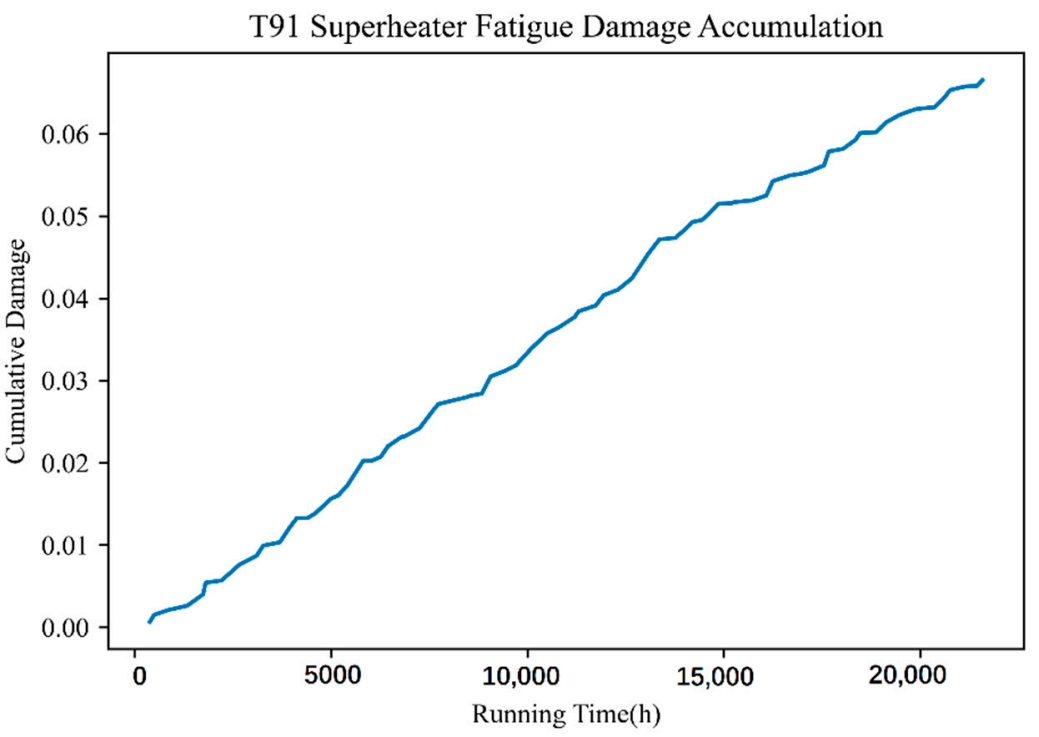

Figure 8. Relationship between service time and fatigue damage of the T91 material in the final superheater stage.

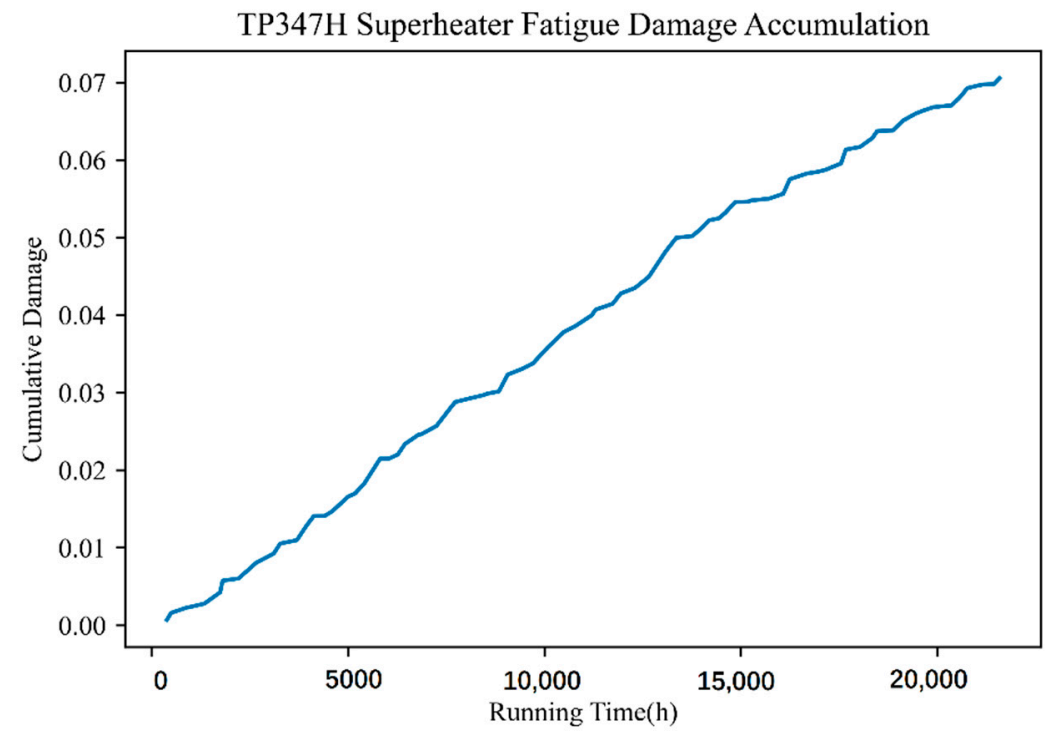

Figure 9. Relationship between service time and fatigue damage of the TP347H material of the final superheater.

\subsection{Calculation of Superheater Creep-Fatigue Interaction Life}

Creep-fatigue interaction damage was significantly promoted, and the occurrence of creep damage also significantly increased the degree of fatigue damage and reduced the 
fatigue life of the material. The results of the creep-fatigue interaction damage prediction model for different materials of the final-stage superheater are illustrated in Figures 10 and 11. Fatigue cracks generated by the accumulation of local damage to the material promote the sprouting of fatigue cracks and then fracture, which is typical of a penetration fracture, while creep damage is due to the collection of internal cavities in the material at grain boundaries to grow into creep cracks, which are typical of fractures along the crystal. The two failure mechanisms are completely different from a microscopic point of view. The fatigue crack and hole in materials will generally undergo significant mutual promotion and development to form a creep-fatigue interaction when a grain-piercing fatigue crack and a hole in the grain boundary meet. However, the traditional physical model ineffectively evaluates the creep-fatigue damage state of the material due to complex operating conditions in the field and failure to calculate real-time fluctuations of operating parameters into the physical model. Thus, the application effect in quantitative assessment of the creep-fatigue interaction damage on the actual service process is poor. This study remarkably improved the accuracy of creep-fatigue damage prediction during actual service by analyzing and mining operational data related to creep-fatigue damage during the evaluation of components in service. The results can help ensure the safe and reliable operation of pressurized components of power station boilers.

T91 Superheater Creep/Fatigue

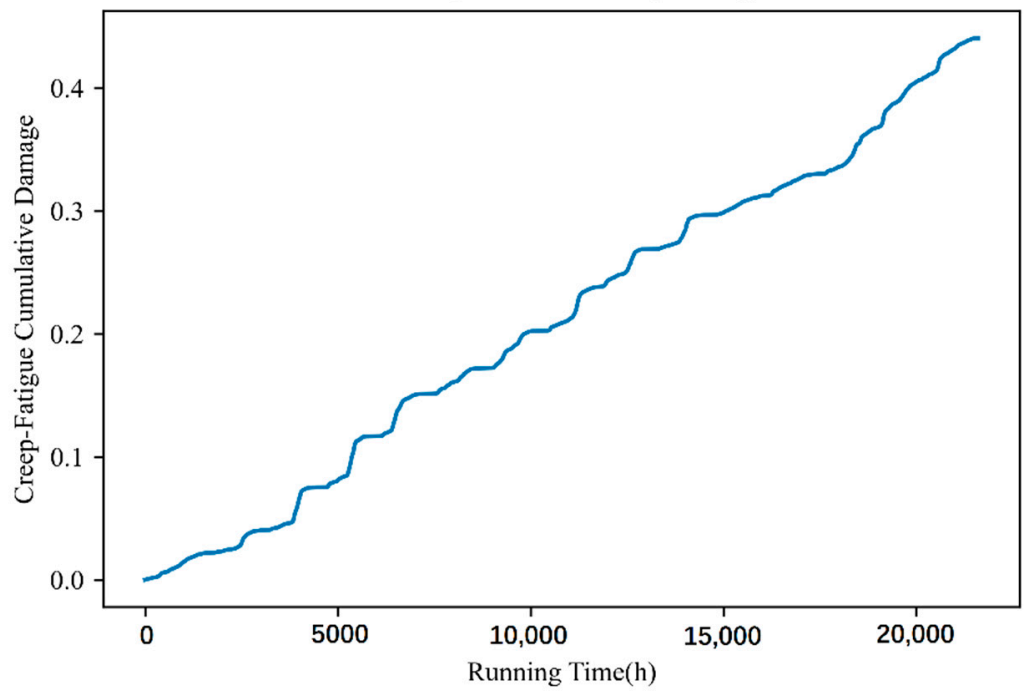

Figure 10. Relationship of creep-fatigue life and damage amount of final superheater T91 material.

\subsection{Reliability Study of the Final-Stage Superheater System}

Using all inspection methods to ensure the reliability of the system is very unrealistic due to the large number of superheater tubes in the final stage. Traditional safety assurance measures for power station boilers in service use a small sample of data combined with expert experience to assess the safety condition of the entire tube system. Although traditional methods can guarantee the reliability of the system to a certain extent, subjective judgment typically involved in such strategies fails to reflect the safety condition of longterm service equipment. Therefore, using a method that can construct a Weibull distribution model of the tube system by inference from small sample data is important. Figure 12 shows the diagram of the calculated results of the Weibull distribution model of the final superheater system of the power station boiler. We can effectively evaluate the current increase in the risk to tubes of the final superheater system in the future and the time point of intensive failure and then prioritize the planned maintenance of the high-risk system on the basis of the risk situation and consequences of failure to avoid unplanned downtime, thus reducing operating costs of the power plant effectively by predicting the probability distribution and density of the failure risk to tubes of the final superheater system. 


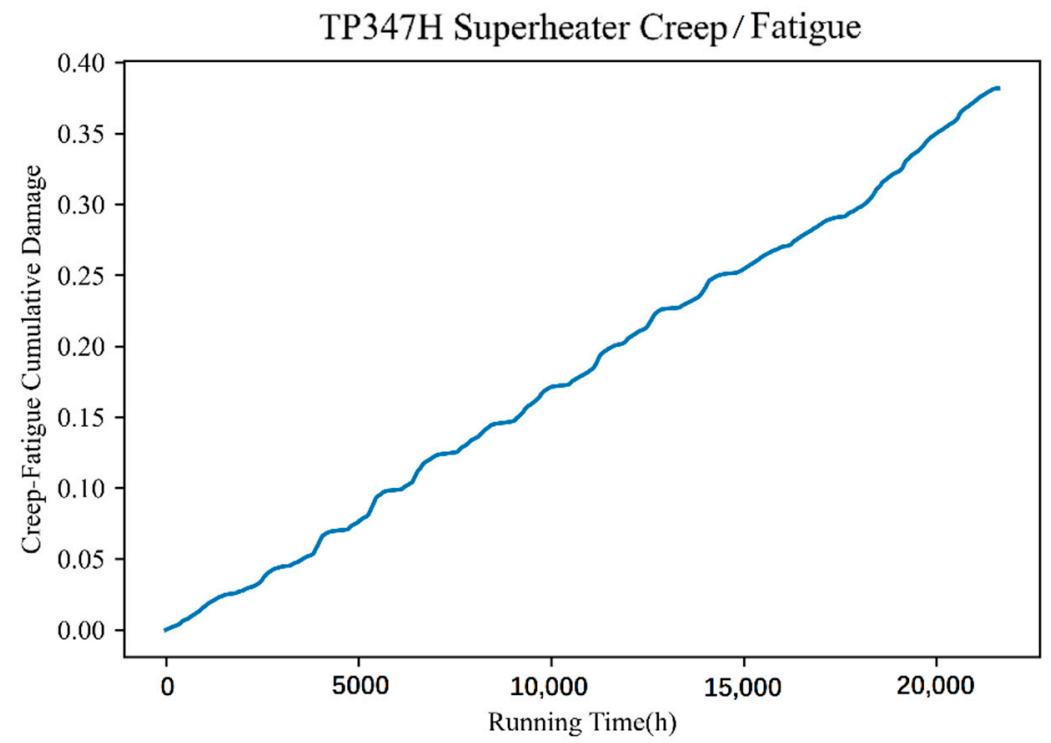

Figure 11. Relationship of creep-fatigue life and damage amount of final superheater TP347H material.

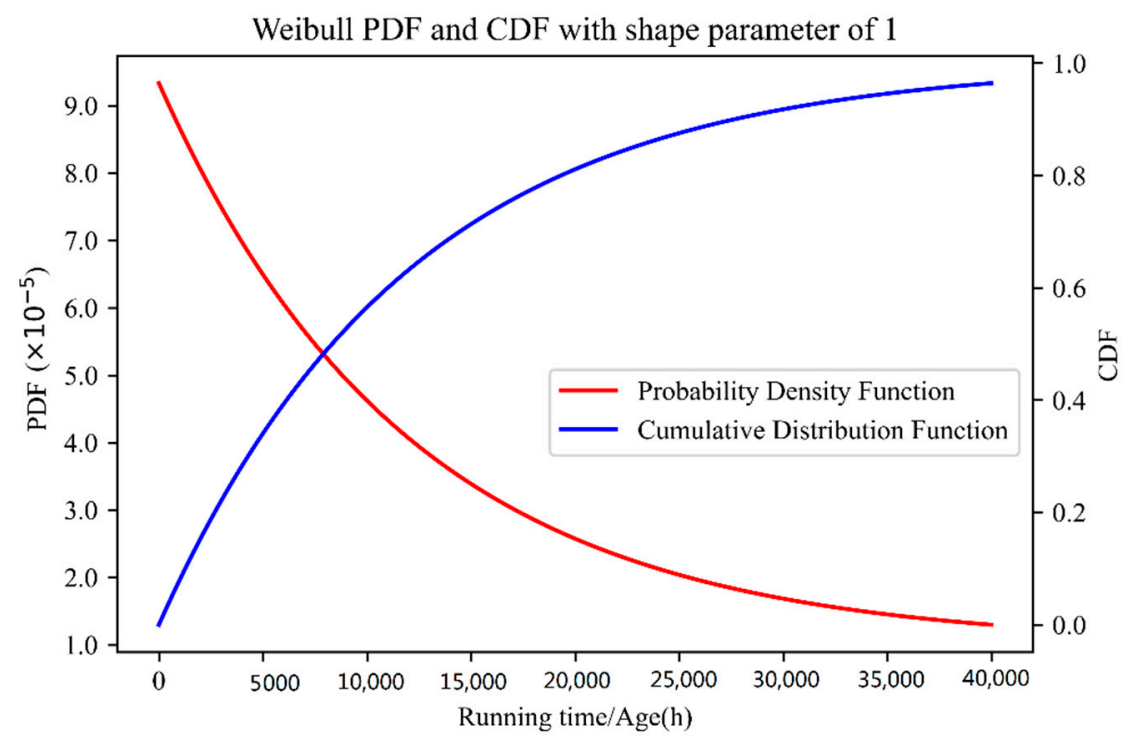

Figure 12. Diagram of the tube Weibull distribution model for the final-stage superheater system.

Figure 13 presents the failure risk of T91 material in the final superheater system of the power station boiler and the remaining life prediction results under various damage modes. Combined with the Weibull distribution model, the current safety condition of pressurized parts of power station boiler was evaluated and the prediction of the future safety condition was predicatively investigated and judged after setting an appropriate threshold to obtain the accumulated failure risk change trend, achieve early warning, avoid accidents effectively, and ensure the economic and safe operation of power station boilers. 

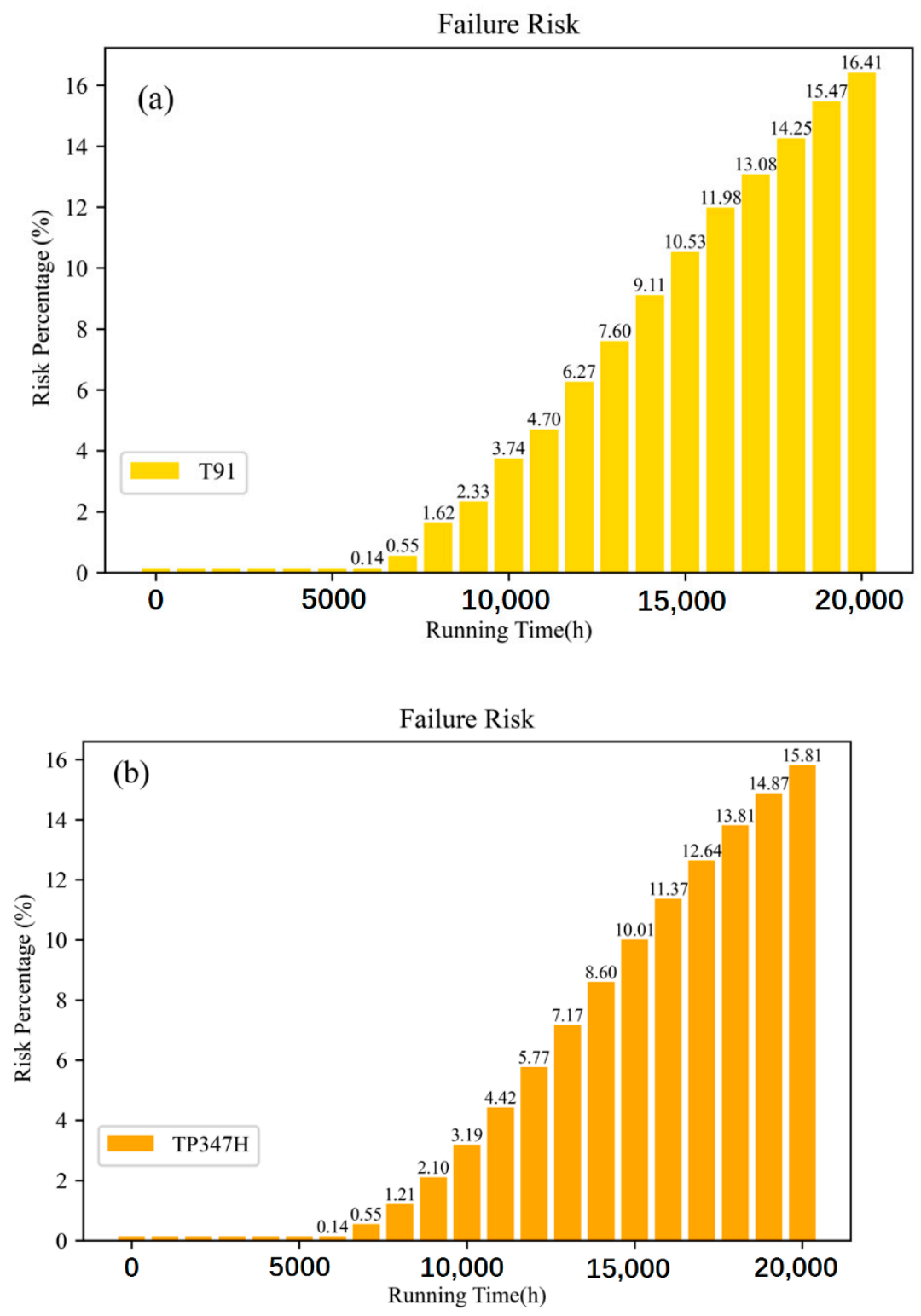

Figure 13. Material risk and remaining life trend prediction of the final-stage superheater system. (a) T91 material. (b) TP347H material.

Figure 13a,b illustrates the use of the Weibull distribution model to achieve a more realistic small sample of inspection data to analyze the risk of the whole system, which was the same for both materials; at the same time, the engineer applied them in the right place according to the differences in service conditions when designing the selection of materials for the heated surfaces (where the main considerations are temperature, pressure, stress). This is why the overall trend of cumulative damage over time was essentially the same for the same damage for the different materials in Figures 6-11, and the analysis of the available base data showed that the risks for T91 and TP347H were also essentially similar.

\section{Conclusions}

Multisource heterogeneous data generated from the actual service of power plants combined with deep learning techniques were used in this study to investigate the direction of creep, fatigue, and coupled creep-fatigue damage characteristics of key pressure-bearing component materials of power plant boilers and their remaining life prediction as well as an early warning of pressure-bearing components. The current increase in the future 
risk to tubes of the final superheater system can be effectively evaluated by predicting the probability distribution and density results of the failure risk of tubes of the final superheater system. Combined with the Weibull distribution model, the current safety condition of pressurized components of the power station boiler can be evaluated and the future safety condition can be predicted to obtain the trend of the accumulated failure risk. This paper used deep learning techniques to explore the patterns that existed in the data when the equipment was in operation, predicting future operating states by learning patterns from historical data. The predicted state data was combined with models in mechanics and materials science. Damage during operation of the equipment and risk conditions were assessed. The combination of data-driven machine learning methods, with knowledge-driven physical models, extends the application of physical models and also provides a fundamental exploration of patterns in equipment operation.

Author Contributions: Data curation, X.Y. (Xu Yang); Formal analysis, X.Y.; Investigation, X.Y. (Xisheng Yang); Project administration, K.L.; Resources, D.A.; Software, X.Y. (Xu Yang); Supervision, G.L.; Validation, D.W.; Writing—original draft, G.L.; Writing—review \& editing, X.R. All authors have read and agreed to the published version of the manuscript.

Funding: This work has been financed by the National Key R \& D Program of China (Grant No. 2018YFC0809004).

Conflicts of Interest: The authors declare no conflict of interest.

\section{References}

1. Yu, F.; Chen, J.; Sun, F.; Zeng, S.; Wang, C. Trend of Technology Innovation in China's Coal-fired electricity industry under resource and environmental constraints. Energy Policy 2011, 39, 1586-1599. [CrossRef]

2. $\quad$ Lin, F.S.; Wang, Z.Z.; Wang, B.Z.; Cheng, S.C.; Xie, X.S. The recent status and development of heat resistant steels and alloys for power plants in China. In Proceedings of the 2009 Symposium on Advanced Power Plant Heat Resistant Steels and Alloys, Shanghai, China, 16 June 2009.

3. Manyika, J.; Chui, M.; Brown, B.; Bughin, J.; Byers, A.H. Big Data: The Next Frontier for Innovation, Competition, and Productivity; McKinsey Global Institute: Washington, DC, USA, 2011.

4. Zhao, R.; Yan, R.Q.; Chen, Z.H.; Mao, K.Z.; Wang, P.; Robert, X.G. Deep learning and its applications to machine health monitoring. Mech. Syst. Signal Processing 2019, 115, 213-237. [CrossRef]

5. Wang, J.J.; Ma, Y.L.; Zhang, L.B.; Robert, X.G.; Wu, D.Z. Deep learning for smart manufacturing: Methods and applications. J. Manuf. Syst. 2018, 48, 144-156. [CrossRef]

6. Zhang, W.S.; Guo, W.W.; Liu, X.; Liu, Y.; Zhou, J.H.; Li, B.; Lu, Q.H.; Yang, S. LSTM-based analysis of industrial IoT equipment. IEEE Access 2018, 6, 23551-23560. [CrossRef]

7. Lecun, Y.; Boser, B.; Denker, J.S.; Henderson, D.; Howard, R.E.; Hubbard, W.; Jackel, L.D. Backpropagation applied to handwritten zip code recognition. Neural Comput. 1989, 1, 541-551. [CrossRef]

8. He, K.; Zhang, X.; Ren, S.; Sun, J. Deep residual learning for image recognition. In Proceedings of the IEEE Conference on Computer Vision and Pattern Recognition, Seattle, WA, USA, 27-30 June 2016; pp. 770-778.

9. Krizhevsky, A.; Sutskever, I.; Hinton, G.E. Imagenet classification with deep convolutional neural networks. Adv. Neural Inf. Processing Syst. 2012, 25, 1097-1105. [CrossRef]

10. Simonyan, K.; Zisserman, A. Very deep convolutional networks for large-scale image recognition. arXiv 2014, arXiv:1409.1556.

11. Hochreiter, S.; Schmidhuber, J. Long Short-Term Memory. Neural Comput. 1997, 9, 1735-1780. [CrossRef]

12. Sagheer, A.; Kotb, M. Time series forecasting of petroleum production using deep LSTM recurrent networks. Neurocomputing 2019, 323, 203-213. [CrossRef]

13. Wu, J.; Hu, K.; Cheng, Y.; Zhu, H.; Shao, X.; Wang, Y. Data-driven remaining useful life prediction via multiple sensor signals and deep long short-term memory neural network. ISA Trans. 2019, 97, 241-250. [CrossRef]

14. Okoh, C.; Rajkumar, R.; Jörn, M.; Redding, L. Overview of Remaining Useful Life Prediction Techniques in Through-life Engineering Services. Procedia CIRP 2014, 16, 158-163. [CrossRef]

15. Rahhal, J.S.; Abualnadi, D. IOT Based Predictive Maintenance Using LSTM RNN Estimator. In Proceedings of the 2020 International Conference on Electrical, Communication, and Computer Engineering ICECCE, Istanbul, Turkey, 12 June 2020; pp. $1-5$.

16. Wang, Y.D.; Addepalli, S.; Zhao, Y.F. Recurrent Neural Networks and its variants in Remaining Useful Life prediction. IFAC-Pap. 2020, 53, 137-142. [CrossRef]

17. Wu, Y.T.; Mei, Y.; Dong, S.P.; Li, L.; Liu, Y.Q. Remaining useful life estimation of engineered systems using vanilla LSTM neural networks. Neurocomputing 2018, 275, 167-179. [CrossRef] 
18. Wang, T.Y.; Henry, L.; Zhao, J.; Wang, W. Multiseries featural LSTM for partial periodic time-series prediction: A case study for steel industry. IEEE Trans. Instrum. Meas. 2020, 69, 5994-6003. [CrossRef]

19. Mateus, B.C.; Mateus, M.; José, T.F.; António, M.C. Anticipating Future Behavior of an Industrial Press Using LSTM Networks. Appl. Sci. 2021, 13, 6101. [CrossRef]

20. Hu, Y.; Sun, X.; Nie, X.; Li, Y.; Liu, L. An enhanced LSTM for trend following of time series. IEEE Access 2019, 7, 34020-34030. [CrossRef]

21. Siami-Namini, S.; Tavakoli, N.; Siami-Namini, A. The Performance of LSTM and BiLSTM in Forecasting Time Series. In Proceedings of the 2019 IEEE International Conference on Big Data, Los Angeles, CA, USA, 9 December 2019; pp. $3285-3292$.

22. Santos, D.; Rosas, F.H.; Leila, W.C.; Silva, D.; Ana, P.B.S. Forecast of multivariate time series sampled from industrial machinery sensors. Braz. J. Oper. Prod. Manag. 2020, 17, 1-12. [CrossRef]

23. Khodabakhsh, A.; Ismail, A.; Bakır, M.; Alagoz, S.M. Forecasting multivariate time-series data using LSTM and mini-batches. In Proceedings of the 7th International Conference on Contemporary Issues in Data Science; Springer: Cham, Switzerland, 2019; pp. 121-129.

24. Werbos, P.J. Backpropagation Through Time: What It Does and How to Do It. Proc. IEEE 1990, 78, 1550-1560. [CrossRef]

25. Liu, Z.; Meng, X.; Wei, H.; Chen, L.; Lu, B.; Wang, Z.; Chen, L. A regularized LSTM method for predicting remaining useful life of rolling bearings. Int. J. Autom. Comput. 2021, 18, 581-593. [CrossRef]

26. Meng, M.; Zhu, M. Deep-convolution-based LSTM network for remaining useful life prediction. IEEE Trans. Ind. Inform. 2020, 17, 1658-1667.

27. Zamrik, S.Y. Damage models for creep-fatigue interaction. In Technology for the 90s A Decade of Progress, The ASME Pressure Vessels and Piping Division; Au-Yang, M.K., Ed.; ASME: New York, NY, USA, 1993; Chapter 3; pp. 84-107.

28. Feng, W. Research on Creep-Fatigue Interaction Damage Model and Life Assessments of P91 Steel. Master's Thesis, South West Jiaotong University, Chengdu, China, 2008.

29. Larson, F.R.; Miller, J. A time temperature relationship for rupture and creep stress. Trans. ASME 1952, 74, 765-775.

30. Kececioglu, D.; Sun, F.B. Mixed-Weibull Parameter Estimation for Burn-in Data Using the Bayesian Approach. Electron. Reliab. 1994, 34, 1657-1679. [CrossRef]

31. Kececioglu, D. Reliability and Life Testing Handbook; PTR Prentice Hall: Hoboken, NJ, USA, 1993; Volume 1.

32. Jiang, R. Failure Models Involving Two Weibull Distributions. Ph.D. Thesis, The University of Queensland, Brisbane, Australia, 1996.

33. Aghajani, A.; Somsen, C.; Eggeler, G. On the effect of long-term creep on the microstructure of a $12 \%$ chromium tempered martensite ferritic steel. Acta Mater. 2009, 57, 5093-5106. [CrossRef]

34. Pesicka, J.; Aghajani, A.; Somsen, C.; Hartmaier, A.; Eggeler, G. How dislocation substructure evolve during long-term creep of a 12\% Cr tempered martensitic ferritic steel. Scr. Mater. 2010, 62, 353-356. [CrossRef] 